\title{
The Influence of Negative Life Events on Suicide Ideation of College Students: Mediating Effect of Cognitive Style
}

\author{
Liang Yingnan ${ }^{1, *}$ Yang Lizhu ${ }^{2}$
}

\author{
${ }^{1}$ Department of basic teaching, Dalian Polytechnic University, Dalian, Liaoning, China \\ ${ }^{2}$ College of psychology, Liaoning Normal University, Dalian, Liaoning, China \\ *Corresponding author. Email: liang_ying_nan@163.com
}

\begin{abstract}
Objective: Investigate the influence of negative life events and cognitive style on suicide ideation of college students. Methods:1617 college students were investigated with positive and negative Suicide Ideation Questionnaire, dysfunctional attitude questionnaire and adolescent life events scale. Results: (1) Negative life events have a direct impact on College Students' suicidal ideation. (2) Cognitive style has a direct impact on College Students' suicidal ideation. (3) Cognitive style plays a mediating role between negative life events and suicidal ideation. Conclusions: Negative life events indirectly affect suicidal ideation through cognitive style. Irrational cognitive style is the mediating factor between negative life events and suicidal ideation.
\end{abstract}

Keywords: Suicidal ideation, negative life events, cognitive style, mediating effect

\section{INTRODUCTION}

Suicidal ideation is the thought that an individual wants to die, but does not put it into action. It is an important part and necessary stage of suicidal behavior [1]. The formation of suicidal ideation involves many factors. In the existing studies, only one factor is focused on the relationship between suicidal ideation and suicidal ideation. For example, it involves negative life events and suicidal ideation[2,3], personality and suicidal ideation[4,5], Cognitive style and suicidal ideation[6], etc.Most of them have only done relevant research, and the possible causal relationship is only limited to speculation and lack of further confirmatory research. The research is relatively scattered, and it is rare to comprehensively and systematically investigate the relationship between them and suicidal ideation. From the perspective of ecosystem development, it is necessary to explore the relationship between these factors as a whole. According to the theory of stress susceptibility model, suicide ideation and behavior are the process of interaction among stress factors (including negative life events, environment, etc.), protective factors (including family, society, culture, etc.) and quality factors (including personality, cognition, etc.) [7]. Quality factor is considered to be extremely important, that is, regardless of the nature of stress, quality factor may lead to suicidal behavior. Life events and situations that induce stress play the role of trigger. When individuals experience stress under the action of life events, the quality base of individual susceptibility is activated. Repeated and lasting trauma can increase people's vulnerability, and then damage their coping ability. In order to reduce the severity of stress, they increase suicide ideation. Therefore, on the basis of this theoretical model, this study will take negative life events and cognitive style as the breakthrough point to investigate the influence of negative life events and cognitive style on College Students' suicide ideation. This can not only enrich the theoretical research system of the mechanism of suicidal ideation, but also has a strong practical significance for the prevention and intervention of College Students' suicidal ideation.

\section{RESEARCH OBJECTS AND METHODS}

\subsection{Sample}

Taking the class as the unit of cluster random sampling method, 1800 students aged 18-25 in 60 classes of grade 1-5 from four universities in Northeast China were selected. A total of 1800 questionnaires were 
distributed and 1800 were returned. There were 1617 valid questionnaires, and the effective rate was $89.83 \%$, including 762 boys, 855 girls, 364 in grade one, 350 in grade two, 349 in grade three, 329 in grade four and 225 in grade five.

\subsection{Measures}

\subsubsection{Positive and negative Suicidal Ideation Questionnaire (Pansi)}

A self-evaluation questionnaire was developed by Osman and his colleagues in 1998 to evaluate the suicidal ideation. The questionnaire consists of two dimensions: positive ideation (pansi-pi) (6 items) and negative ideation (pansi-ni) (8 items). According to the situation in the past two weeks, the score standard is 5 points. Later, Osman and his colleagues conducted a series of studies using the Pansi questionnaire as a tool, which showed that the questionnaire had good reliability and validity [8]

\subsubsection{Dysfunctional attitude questionnaire (}

\section{$D A S)$}

The questionnaire consists of eight factors, including vulnerability, attraction and exclusion, perfection, compulsion, seeking approval, dependence, autonomous attitude and cognitive philosophy. The higher the score, the more distorted the cognition. The scoring standard is 7 points. The internal consistency reliability of the total questionnaire was 0.88 , and the test-retest reliability was 0.84 . The total score of DAS and the scores of each dimension were moderately correlated with the corresponding BDI total score [9].

\subsubsection{Adolescent life events scale (ASLEC)}

ASLEC is a self-rated questionnaire, which consists of 27 negative life events that may bring psychological reactions. There are six factors: interpersonal relationship factor (I), learning stress factor (II), punishment factor (III), loss factor (IV), health adaptation factor (V) and other factors (VI) to summarize and analyze which factor has a high average score, which indicates that the incidence of negative life events in this item is high, and the psychological pressure caused by it is high. The Cronbach a coefficient and split half reliability coefficient of ASLEC were 0.85 and 0.88 respectively. The total score of ASLEC was positively correlated with negative coping score $(\mathrm{r}=0.31)$ and locus of control score $(r=0.22)$ in cnsie. In addition, the total score of ASLEC had a significant predictive effect on the scores of self rating Anxiety Scale (SAS) $(b=0.29)$ and self rating Depression Scale (SDS) $(b=0.20)[10]$.

\subsection{Procedures}

The group test was conducted in class, and the scale was paper and pen questionnaire. Before the test, explain the intention to the students, read out the instruction, and then start the test after all the students know the test procedure. The questionnaire was collected immediately after the test.

\subsection{Data Analysis}

SPSS25.0 was used to analyze the data.

\section{RESULTS}

This study constructs the mediating effect relationship among college students' negative life events, cognitive style and suicidal ideation (see Figure 1). Negative life events were the independent variable, cognitive style was the intermediary variable, and suicidal ideation was the dependent variable. Statistical analysis was conducted according to the mediating effect test procedure[11].

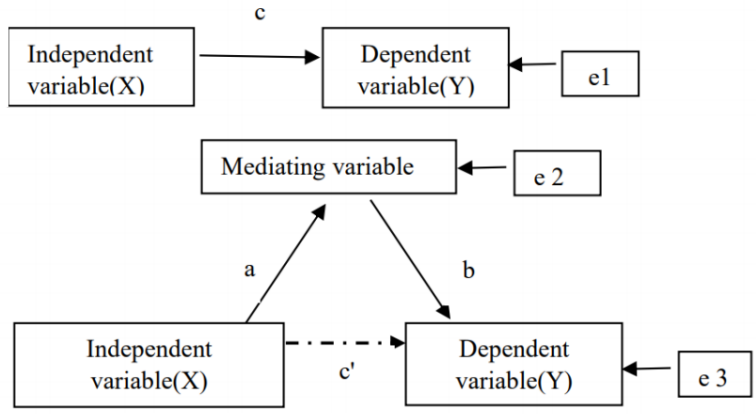

Figure 1 Mediating effect diagram

Table 1 The mediating effect of cognitive style

\begin{tabular}{llccl}
\hline \multirow{2}{*}{$\begin{array}{l}\text { dependent } \\
\text { variable }\end{array}$} & \multicolumn{1}{c}{$\begin{array}{c}\text { The } \\
\text { First step }\end{array}$} & Test c & \\
\cline { 2 - 4 } & \multicolumn{1}{c}{$B$} & $S E$ & $\beta$ \\
\hline Suicidal ideation & 0.071 & 0.002 & 0.630 & $32.564^{* * *}$ \\
\hline
\end{tabular}

Notes : ${ }^{* * *} P<0.001$ 
Table 2 The mediating effect of cognitive style

\begin{tabular}{lcccc}
\hline \multirow{2}{*}{ dependent variable } & $\begin{array}{c}\text { The } \\
\text { second step }\end{array}$ & Test a & & \multirow{2}{*}{$t$} \\
\cline { 2 - 4 } & $B$ & $S E$ & $\beta$ & \\
\hline Suicidal ideation & 0.585 & 0.034 & 0.396 & $17.317^{* * *}$ \\
\hline
\end{tabular}

Notes : ${ }^{* * *} P<0.001$

Table 3 The mediating effect of cognitive style

\begin{tabular}{lcccc}
\hline \multirow{2}{*}{ dependent variable } & \multicolumn{2}{c}{ The } & Test b & \\
& third step & $S E$ & $\beta$ & $t$ \\
\cline { 2 - 4 } & $B$ & & & \\
\hline Suicidal ideation & 0.023 & 0.002 & 0.296 & $14.977^{* * * *}$ \\
& & & \\
\hline
\end{tabular}

Notes: ${ }^{* * *} P<0.001$

Table 4 The mediating effect of cognitive style

\begin{tabular}{|c|c|c|c|c|c|}
\hline \multirow{2}{*}{ dependent variable } & $\begin{array}{l}\text { The } \\
\text { Fourth step }\end{array}$ & \multicolumn{3}{|c|}{ Test c' } & \multirow{2}{*}{$t$} \\
\hline & $B$ & $S E$ & $S E$ & $\beta$ & \\
\hline Suicidal ideation & 0.058 & 0.002 & & 0.513 & $25.979^{* * *}$ \\
\hline
\end{tabular}

Notes : ${ }^{* * * *} P<0.001$

Firstly, the item scores of independent variables (negative life events), mediating variables (cognitive style) and dependent variables (suicide ideation) were combined to get the mean value and centralization (the mean deviation after the item mean value) to get the centralization of negative life events, cognitive style and suicide ideation.

Secondly, according to Wen Zhonglin's intermediate test procedure, the first step is to test whether the $\mathbf{c}$ in the equation $y=c x+e$ is significant. From table 1 , we can see that the regression effect of equation $y=c x+e$ is significant, and the $\mathbf{c}$ value is 0.630 , and the significance is $p<0.001$. We can test the significance of equation $\mathrm{m}=\mathrm{ax}+\mathrm{e}$.

Thirdly, the significance of a was tested according to Wen Zhonglin's second step test procedure. According to the result analysis in Table 2 , in the equation $\mathrm{m}=\mathrm{ax}+\mathrm{e}$, the value of a was 0.396 , and the significance was $p<0.001$.

Finally, the significance of $\mathbf{b}$ and $\mathbf{c}^{\prime}$ is tested at the same time. From table 3 and table 4 , in the equation $\mathrm{y}=\mathrm{c}^{\prime} \mathrm{x}+\mathrm{bm}+\mathrm{e}, \mathbf{b}$ value is 0.296 , the significance is $p<$
0.001 , $\mathbf{c}^{\prime}$ value is 0.513 , the significance is $p<0.001$. Therefore, there is a partial mediating effect. The mediating effect of independent variables on dependent variables is not completely mediated by the mediating variable cognitive style. Negative life events have a direct effect on suicidal ideation The ratio of mediating effect to total effect is

effect $_{\mathrm{m}}=\mathrm{ab} / \mathrm{c}=0.396 \times 0.296 / 0.630=0.186, \quad$ cognitive style has a partial mediating effect on the impact of negative life events on suicidal ideation, and the ratio of mediating effect to total effect is 0.186 .

\section{DISSCUSSION AND CONCLUSION}

\subsection{Negative life events have a direct impact on suicidal ideation.}

The results show that negative life events have a direct impact on College Students' suicidal ideation, and negative life events are an important factor in college students' suicidal ideation. Casey et al. Pointed out that the risk factors of suicidal ideation include negative life 
events [12]. Hu Zhihong and Guo Qiang showed that the score of negative life events in suicide ideation group was significantly higher than that in non suicide ideation group, suggesting that the occurrence of suicide ideation is related to negative life events [13]. According to the biological theory of suicidal ideation, "adverse life events cause people's psychological pressure and destroy the stability of the human body's internal environment. Strong and lasting stress can lead to physiological and psychological disorders, and even mental disorders and physical diseases. On the basis of a certain personality tendency, psychological disease symptoms are produced, which plays a" trigger "role in suicidal ideation" [14].

College Students' suicidal ideation is often related to stressful life events. Stressful life events are the direct causes of their mental diseases, such as freshmen's enrollment, seniors' graduation, study pressure, employment problems, physical diseases, emotional problems, etc. Among them, the pressure of study and employment has the greatest impact on College Students' suicidal ideation. Lovelorn is also one of the main reasons for college students' suicidal ideation.

\subsection{Cognitive style have a direct impact on suicidal ideation.}

The results show that cognitive style also has a direct impact on College Students' suicidal ideation, and irrational cognitive style is an important reason for college students' suicidal ideation. Although the selfawareness of college students has gradually enhanced, the level of self-awareness has also been significantly improved, and the ability of independent thinking and problem-solving has also been developed, there are still some deficiencies in social experience, knowledge level, cognitive level and so on, which makes it inevitable for college students to consider problems more subjectively and cannot objectively and comprehensively treat people and things. Therefore, when faced with specific problems, often only see the negative aspects of the problem, and thus deny themselves, and eventually lose confidence in life.

\subsection{Negative life events indirectly affect suicidal ideation through cognitive style. cognitive style is the mediating factor between negative life events and suicidal ideation.}

The results show that suicidal ideation is directly or indirectly influenced by many factors. Negative life events and cognitive style can directly predict suicidal ideation, indicating that individual specific psychological characteristics, such as cognitive style bias or encountering negative life events, can directly lead to suicidal ideation. At the same time, negative life events also have an indirect impact on suicidal ideation through cognitive style. Nowadays, college students will inevitably encounter some pressure and setbacks in life. The key lies in their own views and attitudes towards things, that is, their own cognitive style. Reasonable cognitive style will weaken the generation of suicidal ideation, while unreasonable cognitive style will increase the generation of suicidal ideation. Therefore, negative life events are only the external cause of College Students' suicidal ideation, and the real effect is their own individual psychological characteristics. See Figure 2.

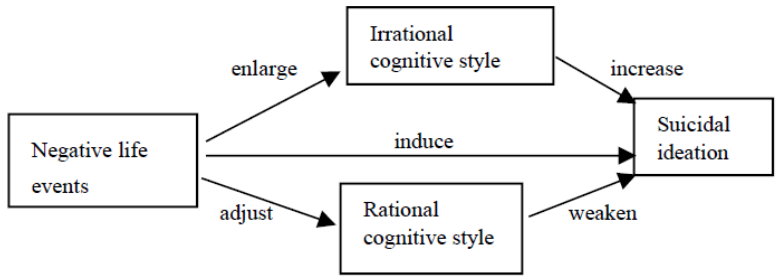

Figure 2 The influence model of negative life events and cognitive style on suicidal ideation

The results show that irrational cognitive style is the mediating factor between negative life events and suicidal ideation. Cognitive bias is an important internal factor of College Students' suicidal ideation. The theory of self avoidance points out that the generation of suicidal ideation is closely related to inappropriate attribution and cognitive characteristics [15]. Beck further classified dysfunctional attitudes into three categories, namely achievement (need to succeed, high operating standards), acceptance (be liked, be loved) and control (to control the development and change of things, to be strong, etc.). This potential dysfunctional hypothesis or schema is the basis for people to evaluate life events, endow experience facts with special significance, and dominate the way people deal with things. The potential dysfunctional attitude of suicidal ideation can be activated by some stressful life event. Once activated, a large number of "negative thoughts" will be produced. The content of negative thoughts can be the explanation of current experience, negative expectation of the future, or negative explanation of past events. It is these negative thoughts that lead to suicidal ideation.

\section{AUTHORS' CONTRIBUTIONS}

Y.N.Liang designed the study, performed the research, analysed data, and wrote the paper. L.Z.Yang conceived the central idea.

\section{ACKNOWLEDGMENTS}

This work was supported by a grant from Liaoning Province "million talents project" science and technology activities project (Category $\mathrm{C}$ project). 


\section{REFERENCES}

[1] Y.Hu.The influence of College Students' life values on suicide ideation (Eds.), Doctoral Dissertation of Dalian University of Technology, 2015

[2] C.L.Bakhiyi,I.Jaussent,S.Beziat, etal .Positive and negative life events and reasons for living modulate suicidal ideation in a sample of patients with history of suicide attempts (Eds.),Journal of Psychiatric Research,Vol.88,2017,pp.64-71.DOI: https://doi.org/10.1016/j.jpsychires.2016.12.022

[3] X.Z. Chen,Z.R.Jia. X.J.Yang.Effects of life events, self esteem and depression on suicidal ideation of College Students(Eds.), Chinese Journal of health psychology,Vol.28,2020.pp.123-127.DOI: https://doi.org/10.13342/j.cnki.cjhp.2020.10.027

[4] U.Lewitzka,S.Spirling,D.Ritter, etal . Suicidal Ideation vs. Suicide Attempts: Clinical and Psychosocial Profile Differences Among Depressed Patients: A Study on Personality Traits, Psychopathological Variables, and Sociodemographic Factors in 228 Patients.(Eds.),Journal of Nervous and Mental Disease , Vol 205,2017,pp.361-371. DOI: https://doi.org/10.1097/NMD.0000000000000667

[5] W. T.Zhuang,Y.X.Li,T.Jiang, J.Tang. Relationship between personality characteristics of childhood negative life events and suicidal ideation of College Students (Eds.), Chinese Journal of School Health, Vol.41,2020, pp.100-103. DOI: https://doi.org/10.16835/j.cnki.10009817.2020.02.025

[6] J.P.Stange,J.L.Hamilton,T.A.Burke,etal. Negative cognitive styles synergistically predict suicidal ideation in bipolar spectrum disorders: A 3-year prospective study(Eds.). Psychiatry Research, Vol.226,2015,pp.162-168.DOI:

https://doi.org/10.1016/j.psychres.2014.12.042

[7] J.J.Mann, M.D.Christine,D.Watemaux ,et al. Toward a clinical model of suicidal behavior in Psychiatric Patients(Eds.). Am J psychiatry, Vol. 156,1999,pp.181-189.DOI:

https://doi.org/10.1002/9781118168226.ch4

[8] A.Osman, P.M.Gutienez, J.Jiandani,et al. A preliminary validation of the Positive and Negative Suicide Ideation (PANSI) Inventory with normal adolescent samples(Eds.). Journal of Clinical Psychology, Vol.59,2003, pp.493-512.DOI: https://doi.org/10.1002/jclp.10154

[9] L. Cai, X.Z.Zhu,S.F.Peng et al.Application of dysfunctional attitude questionnaire in adolescents (Eds.). Chinese Journal of clinical psychology,
Vol.18,2010, pp. 31-34. DOI: https://doi.org/10.16128/j.cnki.10053611.2010.02.021

[10] X.D.Wang,X.L.Wang,H.Ma.Handbook of mental health assessment scale.Chinese Journal of mental health Press, 2000.

[11] Z.L.Wen, L.Zhang,J.T.Hou,et al.The procedure of mediating effect test and its application(Eds.), Acta Psychologica Sinica,Vol.5, 2004, pp. 111-117.

[12] P.R.Casey, G.Dunn, B.D.Kelly,et al. Factors associated with suicidal ideation in the general population: five-centre analysis from the ODIN study(Eds.).British Journal of Psychiatry, Vol. 189,2006,pp.410-415.DOI: https://doi.org/10.1192/bjp.bp.105.017368

[13] S.W.Gao, X.L. Liu. Correlation between quality of life and suicidal ideation of college students in Jilin City and analysis of influencing factors of suicidal ideation(Eds.), Modern Preventive Medicine, Vol.47,2020, pp. 126-129.

[14] W.Dannta.trans. M.Li, et al. Suicide: an unnecessary death.China Light Industry Press, 2003.

[15] L.Helene, J.Nemat, A.Mohamed, et al. Suicidal Ideation in Elite Schools: A Test of the Interpersonal Theory and the Escape Theory of Suicide(Eds.). Suicide and Life-Threatening Behavior, Vol.50, 2020,pp.201210.DOI:https://doi.org/10.1111/sltb.12578 